\title{
Formulação de um Iogurte Suplementado com Compostos Probióticos, Prebióticos e Polpa de Açaí
}

\author{
Nayhara C. Espindula ${ }^{1}$, Carlos E. Cardoso ${ }^{2}$ \\ ${ }^{1}$ Universidade Severino Sombra, CECETEN, Curso de Química Industrial, \\ cardoso@uss.br \\ ${ }^{2}$ Universidade Severino Sombra, CECETEN, Curso de Química Industrial, \\ ncaulo@yahoo.com.br
}

Resumo. Visando melhorar a qualidade de vida dos consumidores, estudos no campo da produção de bebidas com características funcionais estão sendo cada vez mais difundidos e a utilização de compostos probióticos como bactérias lácteas e prebióticos como a inulina e a oligofrutose tem sido uma alternativa viável e eficiente. Assim, o objetivo deste trabalho foi determinar, a partir de um planejamento fatorial $3^{k}$ e da metodologia da superfície de resposta, as melhores condições para o desenvolvimento de uma formulação de iogurte suplementado com oligofrutose e inulina e adicionado de polpa de açaí, bem como as características físico-químicas ( $\mathrm{pH}$ e acidez) da melhor formulação obtida. Os resultados obtidos mostraram que a formulação contendo oligofrutose e inulina nas concentrações de $12 \%$ e $2 \%$, respectivamente, forneceram os melhores resultados $(\mathrm{pH}=4,5 \quad e$ acidez=0,66\%), de acordo com o especificado pela legislação.

Palavras-chave: Polpa de Açaí, probióticos, prebióticos. 


\section{Revista TECCEN — volume 3 - número 1 - abril de 2010 - ISSN 1984-0993}

\section{Introdução}

Atualmente, a preocupação com a saúde e o bem-estar vem aumentando, uma vez que o estilo de vida atribulado tem influenciado negativamente o estado nutricional das populações. Assim, cresce a demanda por produtos que ofereçam benefícios nutricionais e que possuam funções biológicas positivas (produtos funcionais).

Os produtos funcionais podem ser formulados a partir da utilização de dois componentes principais, denominados probióticos e prebióticos [RAUD, 2008]. No grupo dos prebióticos destaca-se a inulina, que é abundante nas raízes da chicória, de onde é extraída industrialmente [De BONDT, 2003].

A inulina (Figura 1) é um polissacarídeo de origem vegetal, cuja unidade é constituída de frutose e de uma molécula de glicose terminal e que possui a fórmula geral $\mathrm{C}_{6 \mathrm{n}} \mathrm{H}_{10 \mathrm{n}+2} \mathrm{O}_{5 \mathrm{n}+1}$ (onde $\mathrm{n}$ é o número de unidades que constituem o polissacarídeo). Por não ser digerida pelas enzimas do intestino humano, é considerada uma fibra alimentar solúvel que alcança o cólon e é utilizada pela flora microbiana [GREENWOOD; EARNSHAW, 1997].

$\mathrm{Na}$ indústria, a inulina é utilizada como ingrediente para a preparação de diversos produtos funcionais e o grau de polimerização condiciona a sua funcionalidade. Os polímeros que apresentam menos de dez unidades de frutose são denominados frutooligossacarídeos ou oligofrutoses, sendo utilizados como adoçantes. Aqueles que apresentam mais de dez unidades não apresentam características adoçantes, sendo utilizados para melhorar a textura dos alimentos em substituição as matérias graxas [e BONDT, 2003].

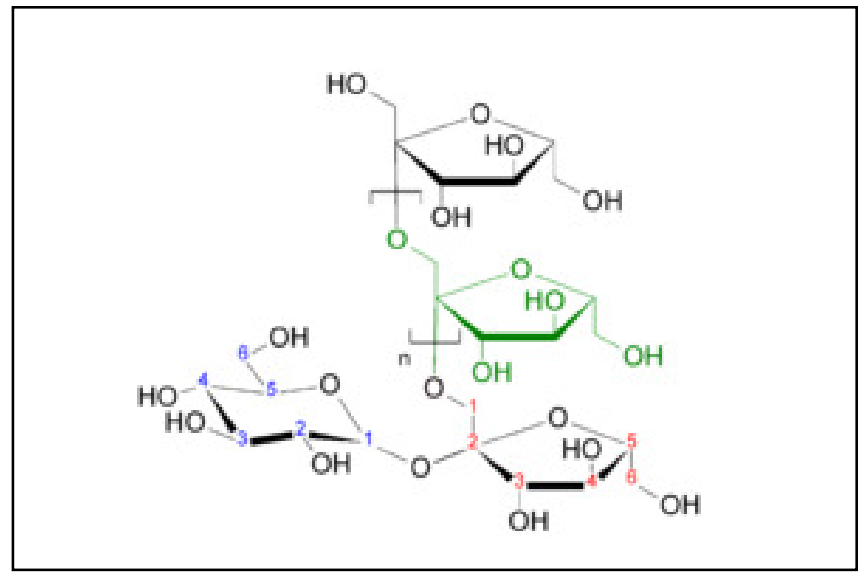

Figura 1. Fórmula estrutural de uma unidade de inulina. Fonte: GREENWOOD; EARNSHAW, 1997.

No grupo dos probióticos estão os microrganismos que afetam beneficamente a flora microbiana intestinal do hospedeiro por promoverem um balanço da microbiota [FUCHS et al, 2005]. Estes participam da fermentação e são considerados um suplemento alimentar constituído de microrganismos vivos, normalmente cepas de 


\section{Revista TECCEN — volume 3 - número 1 - abril de 2010 - ISSN 1984-0993}

lactobacilos e bifidobactérias, que administrados em quantidades adequadas conferem benefícios a saúde [KOMATSU; BURITI; SAAD, 2008].

A adição de inulina e oligofrutose tem por objetivo melhorar as funções biológicas e complementar os benefícios oferecidos pelo probiótico. A inulina e a oligofrutose adicionadas não são consideradas aditivos, pois participam do processo de fermentação. Por ser fermentescível, a inulina permite modificações específicas na composição e/ou na atividade da microbiota gastrintestinal [HAULY; FUCHS; PRUDENCIO-FERREIRA, 2005].

Desta forma, bebidas suplementadas com esses componentes afetam beneficamente uma ou mais funções do organismo e possuem potencial para promover a saúde através de mecanismos não previstos na nutrição convencional [De BONDT, 2003].

De acordo com a Organização das Nações Unidas para Alimentação e Agricultura (FAO),

\footnotetext{
"iogurte é um leite coagulado obtido por fermentação láctica, através da adição de Lactobacillus bulgaricus e Streptococcus thermophilus ao leite, pasteurizado ou concentrado, com ou sem aditivos opcionais. Os microorganismos no produto final precisam ser viáveis e abundantes" (FAO, apud FUCHS et al, 2005).
}

Adicionalmente, Rapacci (1999) e Tenchini de Macedo (2000) dizem que a fermentação do leite deve ser feita procurando equilibrar o crescimento de ambas as bactérias, de modo a se obter um produto suficientemente ácido e aromático. Neste trabalho, o iogurte foi escolhido para suplementação com inulina e oligofrutose devido as suas características nutricionais já conhecidas e a sua grande aceitação no mercado consumidor, além de conter naturalmente o probiótico.

Vários estudos tem demonstrado que a ingestão de quantidades moderadas de inulina e oligofrutose tem como resultado um aumento significativo na quantidade de bifidobactérias benéficas presentes no trato gastrintestinal inferior do ser humano, além de promover uma redução significativa do teor de bactérias indesejáveis [De BONDT, 2003].

Ainda segundo de Bondt (2003), a oligofrutose, por sua vez, é mais solúvel que a inulina e moderadamente doce (cerca de um terço da doçura da sacarose). Assim, pode ser combinada facilmente com um edulcorante para desenvolver um perfil de doçura muito similar ao do açúcar. Em ambos os casos, são fáceis de processar e adicionar a líquidos, podendo ser incorporadas em formulações para oferecer oportunidades de melhoria de produtos.

Tais melhorias podem, ainda, ser obtidas pela adição de polpas de frutas, já que estas possuem, comprovadamente, um elevado valor nutricional. A polpa do açaí é um ótimo energético (cerca de 250 quilocalorias para cada 100 gramas de açaí), além de ser fonte de proteínas, fibras, vitaminas (C, E, B1 e B2), fósforo, ferro, cálcio e antocianinas (flavonóides). Reporta-se, também, que o açaí favorece a circulação do sangue, melhora 


\section{Revista TECCEN — volume 3 - número 1 - abril de 2010 - ISSN 1984-0993}

as funções intestinais, combate os radicais livres e previne a anemia [JARDIM; MOURÃO; GROSSMANN, 2004].

Assim, diante da crescente demanda por alimentos mais saudáveis, capazes de trazer benefícios extras à saúde, e dos potenciais efeitos benéficos de prebióticos, probióticos e do açaí, este trabalho teve como objetivo determinar, utilizando a metodologia da superfície de resposta, as melhores condições para a formulação de um iogurte suplementado com oligofrutose e inulina e adicionado de polpa de açaí.

\section{Experimental}

\subsection{Reagentes e materiais diversos}

Todas as análises foram realizadas com reagentes de grau analítico e água destilada. A água destilada foi obtida de um destilador QUIMIS Modelo Q341.12 [QUIMIS, Brasil].

Os suplementos prebióticos oligofrutose (Raftilose P95) e inulina (Raftiline GR) utilizados para o preparo do iogurte foram fornecidos pelo distribuidor (Clariant Brasil) da empresa BENEO-Orafti. O fermento lácteo BioRich, fabricado pela Chr. Hansen, usado para a inoculação do iogurte é composto de culturas de L. acidophilus $L A-5$ (1,0 x $\left.10^{6} \mathrm{UFC} \mathrm{g}^{-1}\right)$, Bifidobacterium BB-12 $\left(1,0 \times 10^{6} \mathrm{UFC} \mathrm{g}^{-1}\right)$ e S. thermophilus $\quad\left(1,0 \times 10^{6}\right.$ $\mathrm{UFC} \mathrm{g}^{-1}$ ).

Como meio para a inoculação, suplementação e preparo do iogurte foi escolhido o leite integral longa vida (esterilizado). Esta escolha baseou-se nas características físico-químicas que o leite confere ao iogurte, como consistência e paladar. As amostras de leite e de polpa de açaí foram adquiridas no comércio da cidade de Vassouras (RJ), sempre no mesmo revendedor e armazenadas em geladeira a $8^{\circ} \mathrm{C}$ (leite) e $-2^{\circ} \mathrm{C}$ (açaí) por, no máximo, um dia.

\subsection{Procedimentos}

O material utilizado em todos os procedimentos foi previamente lavado com detergente neutro, enxaguado com água destilada e seco em estufa (Estufa de Secagem e Esterilização Mod. 315SE - FANEM, Brasil).

Os percentuais de inulina e oligofrutose foram determinadas a partir da metodologia da superfície de resposta, usando um delineamento fatorial $3^{2}$ completo. Foram realizados 9 ensaios com diferentes concentrações $(\%, \mathrm{~m} / \mathrm{v})$ das variáveis independentes (inulina e oligofrutose) e, nos iogurtes produzidos, analisados o $\mathrm{pH}$ e a acidez titulável (variáveis dependentes). Todas as massas necessárias para o preparo das formulações foram pesadas em balança analítica [COLEMAN Mod. FA2004, Brasil].

Os volumes de leite $(100 \mathrm{~mL})$ foram adicionados em frascos de Duran que foram previamente limpos e expostos a radiação ultra-violeta (UV) por 20 minutos para reduzir a carga microbiana do recipiente. As massas de inulina e oligofrutose foram pesadas e adicionadas cada qual em um recipiente identificado com a respectiva concentração. A solução foi homogeneizada, tampada e sofreu esterilização em autoclave, onde permaneceu por 20 minutos em vapor fluente. Após a esterilização os 


\section{Revista TECCEN — volume 3 - número 1 - abril de 2010 - ISSN 1984-0993}

substratos foram resfriados até a temperatura de $42^{\circ} \mathrm{C}$ e inoculados com fermento lácteo na concentração de $0,12 \%$, tendo permanecido em estufa nessa temperatura por 6 horas. Após o período de fermentação, as formulações ficaram sob refrigeração durante aproximadamente 8 horas. Após a otimização e a determinação das melhores respostas, o iogurte formulado recebeu a adição de $5 \%$ de polpa de açaí para teste de aceitabilidade.

$\mathrm{O}$ valor de $\mathrm{pH}$ das formulações foi obtido ao término do período de fermentação estipulado através de potenciômetro digital (MARTE MB-10, Brasil), previamente calibrado. A acidez foi determinada por titulação com solução de $\mathrm{NaOH} 0,1 \mathrm{~mol} \mathrm{~L}^{-1}$ conforme procedimento do Instituto Adolf Lutz [INSTITUTO ADOLF LUTZ, 1976] e expressa em percentagem de ácido lático. As amostras foram analisadas após o término do processo fermentativo.

\subsection{Aplicativos para tratamento dos dados}

Todos os dados foram manipulados no aplicativo STATISTICA Versão 6.0 (StatSoft, USA). Neste pacote foram também processadas as análises de variância (ANOVA), traçados os gráficos e as superfícies de resposta.

\section{Resultados e Discussões}

A otimização pode ser dividida em estágios que, normalmente, caracterizam-se pela definição da função objetivo (resposta), pela determinação dos fatores (variáveis) que apresentam influências significativas sobre a resposta que se deseja otimizar e pela otimização propriamente dita, isto é, a combinação dos valores dos fatores selecionados que resultem na melhor resposta [EIRAS; CUELBAS; de ANDRADE, 1994].

Um procedimento criterioso para a otimização de um sistema químico deve envolver a realização de experimentos de varredura para caracterizar as variáveis do sistema usando um planejamento fatorial, a localização da região ótima ou ideal e o ajuste fino da região ótima, usando uma superfície de respostas, dependendo de quão apurados se desejam os resultados [BARROS NETO; SCARMÍNIO; BRUNS, 2003].

Alguns cuidados devem ser observados para que se possa obter o máximo de informação na realização do planejamento fatorial. Dentre estes encontra-se a necessidade de realizar repetições de alguns ensaios para que se possa estimar o erro experimental. As replicatas devem ser repetições autênticas, devendo representar adequadamente o espaço experimental no qual o planejamento fatorial foi desenvolvido. Outro cuidado a ser observado refere-se à realização dos experimentos. É importante que todos os ensaios e replicatas previstos no desenvolvimento do fatorial sejam realizados de forma aleatória. Estes cuidados visam evitar distorções estatísticas que possam comprometer a qualidade dos resultados obtidos e dos efeitos calculados para as variáveis estudadas [BARROS NETO; SCARMÍNIO; BRUNS, 2003].

As respostas para os 9 ensaios, expressas em valores médios de três repetições, foram obtidas após um planejamento fatorial $3^{2}$ mostrado na Tabela 1. 


\section{Revista TECCEN — volume 3 - número 1 - abril de 2010 - ISSN 1984-0993}

Tabela 1. Planejamento fatorial $3^{2}$ e respostas para as formulações dos iogurtes suplementados

\begin{tabular}{|c|c|c|c|c|c|}
\hline \multirow{2}{*}{ Experimento } & \multicolumn{3}{|c|}{ Variáveis Codificadas } & \multirow{2}{*}{$\mathrm{pH}$} & \multirow{2}{*}{ Acidez } \\
\hline & $\mathrm{X}_{1}$ & & $\mathrm{X}_{2}$ & & \\
\hline 1 & -1 & & -1 & 4,47 & 0,75 \\
\hline 2 & -1 & & 0 & 4,47 & 0,66 \\
\hline 3 & -1 & & 1 & 4,48 & 0,63 \\
\hline 4 & 0 & & -1 & 4,49 & 0,68 \\
\hline 5 & 0 & & 0 & 4,52 & 0,67 \\
\hline 6 & 0 & & 1 & 4,53 & 0,63 \\
\hline 7 & 1 & & -1 & 4,52 & 0,66 \\
\hline 8 & 1 & & 0 & 4,55 & 0,65 \\
\hline 9 & 1 & & 1 & 4,68 & 0,56 \\
\hline \multirow{2}{*}{ Variáveis Independentes } & \multicolumn{3}{|c|}{ Níveis Codificados } & & \\
\hline & -1 & 0 & 1 & & \\
\hline Oligofrutose (\%) $\mathrm{X}_{1}$ & 12 & 14 & 16 & & \\
\hline Inulina $(\%) \mathrm{X}_{2}$ & 2,5 & 4,5 & 6,5 & & \\
\hline
\end{tabular}

Utilizando-se o software Statistica 6.0, após a entrada adequada dos dados obtém-se a soma quadrática (SQ) do erro, dada pela diferença (Equação 1)

$\mathrm{SQ}_{\text {resíduo }}=\mathrm{SQ}_{\text {Total }}-\mathrm{SQ}_{1}-\mathrm{SQ}_{2}-\mathrm{SQ}_{1 * 2}$

$\mathrm{SQ}_{\text {resíduo }}=0,001975$ (variável pH) e 0,002878 (variável Acidez)

E a estatística F será (Equações 2 a 5)

$\mathrm{F}=\mathrm{S}_{1} / \mathrm{S}_{2}$

Oligofrutose (\%): $\mathrm{F}_{\text {calculado }}=\mathrm{MQ}_{1} / \mathrm{MQ}_{\text {erro }}$

Inulina (\%): $\mathrm{F}_{\text {calculado }}=\mathrm{MQ}_{2} / \mathrm{MQ}_{\text {erro }}$

Interação: $\mathrm{F}_{\text {calculado }}=\mathrm{MQ}_{1 * 2 /} \mathrm{MQ}_{\text {erro }}$

onde,

MQ = Média Quadrática

Os valores calculados são mostrados na Tabela 2. A análise da variância indica haver relevância estatística $(\alpha=5 \%)$ de ambos os fatores (percentual de oligofrutose e percentual de inulina) tanto no valor do $\mathrm{pH}$ quanto na acidez do iogurte, isto é, ambos afetam de maneira significativa o $\mathrm{pH}$ e a acidez da formulação $\left(\mathrm{F}_{\text {calculado }}>\mathrm{F}_{\text {crítico }}\right.$ ) quando um modelo linear (L) é adotado. A interação entre os dois fatores se mostra, 


\section{Revista TECCEN — volume 3 - número 1 - abril de 2010 - ISSN 1984-0993}

também, bastante relevante quanto ao $\mathrm{pH}$ obtido após a formulação e não poderá ser desprezada na região estudada.

Tabela 2. ANOVA - Cálculo da estatística F

\begin{tabular}{lccc}
\hline \multicolumn{1}{c}{ Fonte da variação (var. pH) } & $\mathrm{SQ}$ & $\mathrm{MQ}$ & $\mathrm{F}_{\text {calculado }}$ \\
\hline $\mathrm{X}_{1}$ ) Oligofrutose (\%) (L) & 0,018150 & 0,018150 & 27,56962 \\
Oligofrutose (\%) (Q) & 0,000450 & 0,000450 & 0,68354 \\
$\left.\mathrm{X}_{2}\right)$ Inulina (\%) (L) & 0,007350 & 0,007350 & 11,16456 \\
Inulina (\%) (Q) & 0,000450 & 0,000450 & 0,68354 \\
1L x 2L & 0,005625 & 0,005625 & 8,54430 \\
Erro & 0,001975 & 0,000658 & \\
\hline Fonte da variação (var. Acidez) & $\mathrm{SQ}$ & $\mathrm{MQ}$ & $\mathrm{F}_{\text {calculado }}$ \\
\hline $\left.\mathrm{X}_{1}\right)$ Oligofrutose (\%) (L) & 0,004817 & 0,004817 & 5,02124 \\
Oligofrutose (\%) (Q) & 0,000139 & 0,000139 & 0,14479 \\
(X) Inulina (\%) (L) & 0,012150 & 0,012150 & 12,66602 \\
Inulina (\%) (Q) & 0,000139 & 0,000139 & 0,14479 \\
1L x 2L & 0,000100 & 0,000100 & 0,10425 \\
Erro & 0,002878 & 0,000959 & \\
\hline
\end{tabular}

Analisando-se o gráfico dos resíduos, verificou-se que o modelo construído está adequado (os resíduos obedecem a uma distribuição normal), já que todos os pontos estão colocados na reta ou muito próximos dela (resíduos próximos de zero). Quanto mais próximos os pontos experimentais estiverem da linha contínua, mais será válida a suposição de normalidade dos resíduos. Como os resíduos não estão longe da faixa em questão, descarta-se a presença de outliers, pontos que seriam muito diferentes dos demais dados experimentais e que, após teste específico, poderiam ser excluídos da análise. O gráfico dos resíduos versus valores preditos mostra a aleatoriedade do sistema, indicando que o modelo está ajustado [BARROS NETO; SCARMÍNIO; BRUNS, 2003].

Os coeficientes do modelo de regressão, em termos das variáveis escalonadas são mostrados na Tabela 3. Verifica-se a importância dos efeitos e interações já citadas. Os resultados demonstraram que os interceptos foram significativos $(\mathrm{t}=528,9$ para $\mathrm{pH}$ e $\mathrm{t}=63,4$ para Acidez), indicando que o ponto central para as variáveis independentes foi escolhido adequadamente [BARROS NETO; SCARMÍNIO; BRUNS, 2003]. A partir das estimativas dos coeficientes de regressão, percebeu-se que ambas as variáveis são importantes na formulação do iogurte. $\mathrm{O}$ modelo apresentou um coeficiente de determinação satisfatório $\left(\mathrm{R}^{2}=0,94191\right)$ para a resposta $\mathrm{pH}$ e um coeficiente de determinação mais baixo $\left(\mathrm{R}^{2}=0,85769\right)$ para a variável Acidez. A falta de ajuste não foi significativa, indicando que o modelo pode ser utilizado para fins preditivos [EIRAS; CUELBAS; de ANDRADE, 1994]. 


\section{Revista TECCEN - volume 3 - número 1 - abril de 2010 - ISSN 1984-0993}

Tabela 3. Coeficientes de regressão para as variáveis pH e Acidez

\begin{tabular}{lcccc}
\cline { 2 - 5 } & \multicolumn{2}{c}{$\mathrm{pH}$} & \multicolumn{2}{c}{ Acidez } \\
\cline { 2 - 5 } & Coeficiente & Erro padrão & Coeficiente & Erro padrão \\
\hline Média/Intercepto & 4,523333 & 0,008553 & 0,654444 & 0,010324 \\
$\left(\mathrm{X}_{1}\right)$ Oligofrutose (\%) (L) & 0,055000 & 0,010475 & $-0,028333$ & 0,012644 \\
$\left(\mathrm{X}_{1}\right)$ Oligofrutose (\%) (Q) & $-0,007500$ & 0,009071 & 0,004167 & 0,010950 \\
$\left(\mathrm{X}_{2}\right)$ Inulina (\%) (L) & 0,035000 & 0,010475 & $-0,045000$ & 0,012644 \\
$\left(\mathrm{X}_{2}\right)$ Inulina (\%) (Q) & $-0,007500$ & 0,009071 & 0,004167 & 0,010950 \\
1L x 2L & 0,037500 & 0,012829 & 0,005000 & 0,015486 \\
\hline
\end{tabular}

Os fatores $\mathrm{X}_{1}(\mathrm{Q})$ e $\mathrm{X}_{2}(\mathrm{Q})$ possuem coeficientes negativos, causando decréscimo na resposta $\mathrm{pH}$. Os fatores $\mathrm{X}_{1}(\mathrm{~L})$ e $\mathrm{X}_{2}(\mathrm{~L})$, assim como a interação $1 \mathrm{~L}$ x $2 \mathrm{~L}$ promovem um aumento na variável de resposta $\mathrm{pH}$. Os fatores $\mathrm{X}_{1}(\mathrm{~L})$ e $\mathrm{X}_{2}(\mathrm{~L})$, por sua vez, possuem coeficientes negativos, causando decréscimo na resposta Acidez. Os fatores $\mathrm{X}_{1}(\mathrm{Q})$ e $\mathrm{X}_{2}(\mathrm{Q})$, assim como a interação $1 \mathrm{~L}$ x $2 \mathrm{~L}$ promovem um aumento na variável de resposta Acidez.

No método da análise da superfície de resposta são realizados planejamentos fatoriais, para cujos resultados são ajustados modelos matemáticos. Estas etapas, conhecidas como etapa de deslocamento e modelagem, respectivamente, são repetidas várias vezes, mapeando a superfície de respostas obtidas na direção da região ponto de ótimo desejado. A modelagem normalmente é feita ajustando-se os modelos mais simples, como o linear e o quadrático. Por sua vez, o planejamento fatorial executado geralmente constitui-se de um número pequeno e pré-determinado de experimentos, que são determinados através do ajuste conseguido para o modelo que foi aplicado na etapa imediatamente anterior. Outro detalhe importante é o uso das variáveis em sua forma escalonada, de forma que suas grandezas não interfiram no desenvolvimento do processo de otimização. Os cuidados na realização dos experimentos e de suas replicatas devem ser observados. Usualmente, a modelagem é iniciada utilizando-se o modelo linear.

As equações das superfícies de resposta obtidas em termos das variáveis codificadas serão (Equações 6 e 7):

$\mathrm{pH}=4,52333+0,05500 \mathrm{X}_{1}+0,03500 \mathrm{X}_{2}-0,00750 \mathrm{X}_{1}^{2}-0,00750 \mathrm{X}_{2}^{2}+0,3750 \mathrm{X}_{1} \mathrm{X}_{2}$

Acidez $=0,65444-0,02833 X_{1}-0,04500 X_{2}+0,00416 X_{1}^{2}+0,00416 X_{2}^{2}+0,00500 X_{1} X_{2}$

Adicionalmente, as curvas de nível servem para definir melhor a região de máximo da resposta ( $\mathrm{pH}$ e/ou Acidez). Pode-se identificar, também, os pontos do planejamento em que a resposta parece ser mais sensível à mudança na concentração 


\section{Revista TECCEN — volume 3 - número 1 - abril de 2010 - ISSN 1984-0993}

de oligofrutose do que à mudança da concentração de inulina, fato este já observado pelo maior coeficiente do termo escalonado.

A Figura 2 mostra as superfícies de resposta e as curvas de nível obtidas. De acordo com esta Figura, a formulação contendo $12 \%$ de oligofrutose $\left(\mathrm{X}_{1}\right)$ e $2 \%$ de inulina $\left(\mathrm{X}_{2}\right)$ em 6 horas de fermentação, fornece as melhores condições para a elaboração do iogurte $(\mathrm{pH}=4,5$ e Acidez $=0,70 \%)$. Na prática, o iogurte produzido sob estas condições apresentou pH de 4,2 e acidez de $0,66 \%$, validando o estudo realizado.

As curvas de nível fornecem, ainda, uma análise da tendência de resposta da variável dependente [EIRAS; CUELBAS; de ANDRADE, 1994]. Quando essas linhas possuem curvatura (Figura 2), pode-se dizer que há efeito de interação entre as variáveis colocadas nos eixos. Estas figuras são bastante elucidativas e fornecem informações a respeito das condições de operação do processo. Se maiores valores de $\mathrm{pH}$ forem desejados (região vermelha no gráfico), devem ser utilizados os níveis mais altos das variáveis. 


\section{Revista TECCEN — volume 3 - número 1 - abril de 2010 - ISSN 1984-0993}

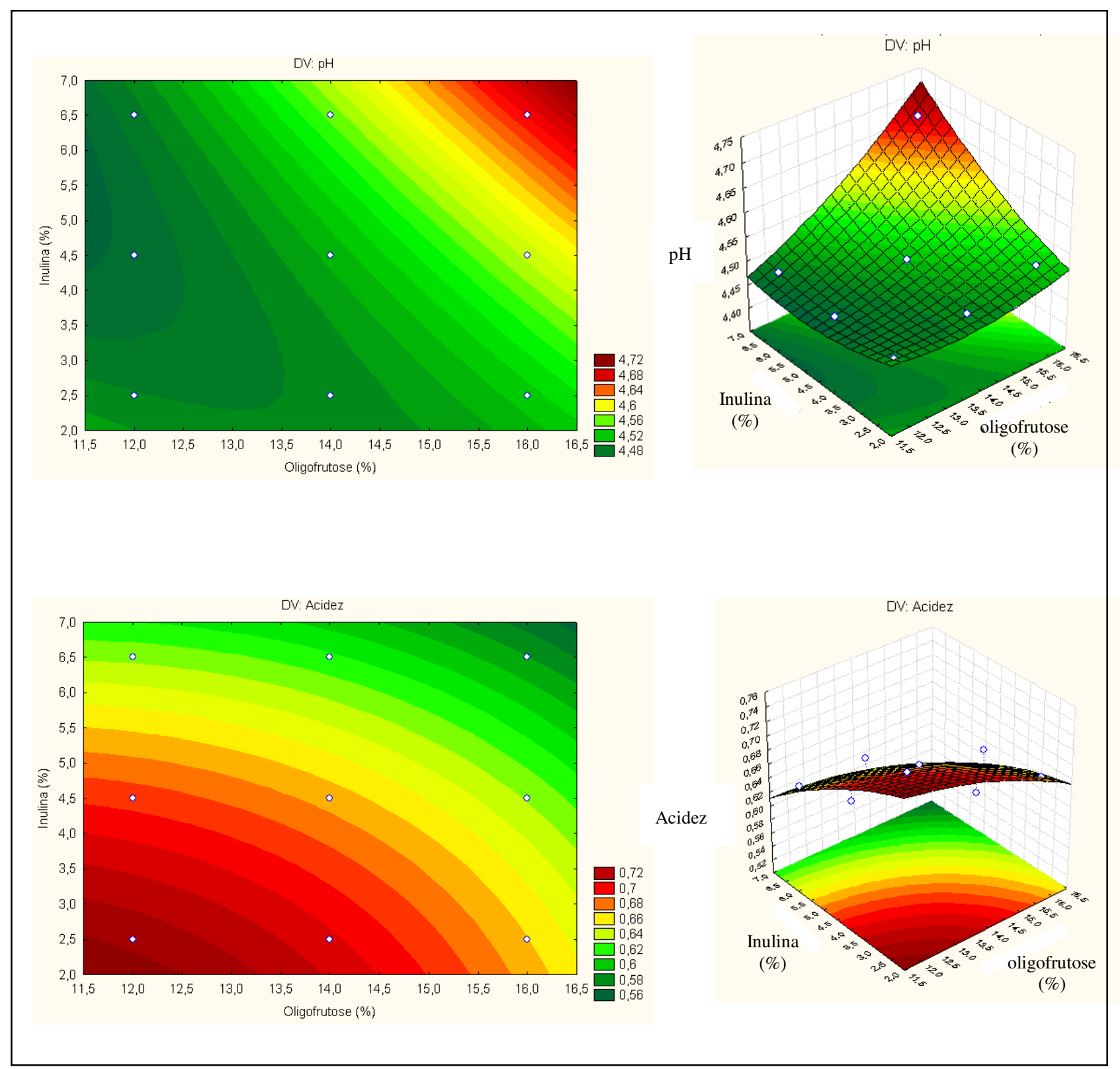

Figura 2. Curvas de Nível e Superfícies de Resposta.

\section{Conclusões}

Na Tabela que apresenta a análise de variância (ANOVA) para esse experimento, utilizou-se o critério $F_{\text {calculado }}>F_{\text {crítico }}$ para rejeitar a hipótese nula (existe evidência de curvatura). Desta forma, comparando-se estes valores para um nível de significância de $5 \%$ pode-se afirmar que os fatores "percentual de oligofrutose" e "percentual de inulina" 


\section{Revista TECCEN — volume 3 - número 1 - abril de 2010 - ISSN 1984-0993}

apresentam efeitos significativos. Isso demonstra que ao menos uma das variáveis do modelo contribui significativamente na aproximação da superfície. A rejeição da hipótese nula também é confirmada porque o valor $\mathrm{p}$ é menor que o nível de significância portanto, o teste é significativo.

Ainda na ANOVA, o teste F, em um nível de significância de 5\%, apresentou existência de uma curvatura no plano da superfície de resposta, havendo evidências, portanto, de que a função não é linear. Ao mesmo tempo, a análise de variância demonstrou que o efeito de interação dos fatores é significativo no modelo.

Com esses resultados, concluiu-se que o modelo de primeira ordem não satisfaz as condições de melhores valores de $\mathrm{pH}$ e Acidez do iogurte com relação às duas variáveis de interesse e, nesse caso, deve-se partir para a construção de um modelo de ordem superior (quadrático, neste caso).

Utilizando-se o método dos mínimos quadrados e a soma quadrática das respostas do experimento, foram construídos os modelos ajustados para as superfícies de resposta consideradas, conforme equações mostradas anteriormente (Equações 6 e 7). Nova análise de variância apontou que a falta de ajuste não é significativa nas superfícies consideradas. $\mathrm{O}$ teste $\mathrm{F}$ no modelo completo mostrou os termos lineares e quadráticos e os efeitos significativos.

A análise da superfície de resposta tridimensional gerada a partir do modelo ajustado permitiu verificar as combinações dos parâmetros dos níveis máximo e mínimo que influenciam o resultado das funções resposta ( $\mathrm{pH}$ e Acidez).

Tornou-se evidente que os 2 fatores que foram considerados no experimento, influenciam as respostas. Com a identificação das principais variáveis foi possível utilizar um procedimento de otimização (metodologia de superfície de resposta), sendo possível modelar a variação da função resposta e, através da análise estatística, foi possível determinar as condições ótimas para a formulação do iogurte suplementado com oligofrutose e inulina ( $2 \%$ de inulina, $12 \%$ de oligofrutose, em $6 \mathrm{~h}$ de fermentação) de acordo com o especificado pela legislação [INDUSTRIA DE LATICINIOS, 1998].

Adicionalmente, o exemplo apresentado neste trabalho mostra que apenas a análise de variância (ANOVA) não é um critério suficiente para a seleção de um modelo, que pode descrever um determinado conjunto de dados, sendo imprescindível que a análise do gráfico dos resíduos também seja feita, mantendo-se uma visão crítica dos resultados obtidos.

\section{Agradecimentos}

Os autores agradecem às empresas BENEO-Orafti e Clariant Brasil que gentilmente cederam os suplementos prebióticos oligofrutose (Raftilose P95) e inulina (Raftiline GR) utilizados neste trabalho. 


\section{Revista TECCEN — volume 3 - número 1 - abril de 2010 -}

ISSN 1984-0993

\section{Referências}

BARROS NETO, B.; SCARMÍNIO, I.S.; BRUNS, R.E., Como fazer experimentos: pesquisa e desenvolvimento na ciência e na indústria, Campinas: Editora Da Unicamp, 2003.

De BONDT, V. Novas tendências para bebidas funcionais, BRASIL ALIMENTOS, 18, Janeiro/Fevereiro, 2003.

EIRAS, S.P.; CUELBAS, C. J.; de ANDRADE, J.C.. Um Estudo Comparativo sobre a Eficiência de Estratégias Quimiométricas de Otimização, Química Nova, 16, 216 219, 1994

FUCHS, R. H. B.; BORSATO. D.; BONA. E.; HAULY M. C. O., Iogurte de soja suplementado com oligofrutose e inulina. Ciênc. Tecnol. Aliment, Campinas, p. 175181, jan./mar., 2005

GREENWOOD, N. N., EARnSHAW, A., Chemistry of the Elements, $2^{\text {nd }}$ ed., Oxford, UK: Butterworth-Heinemann, 1997.

HAULY. M. C. O.; FUCHS R. H. B.; PRUDENCIO-FERREIRA. S. H. Suplementação de iogurte de soja com frutooligossacarídeos:características probióticas e aceitabilidade. Rev. Nutr., Campinas, p. 613-622, set./out., 2005.

INSTITUTO ADOLF LUTZ. Normas analíticas do Instituto Adolf Lutz. São Paulo: Secretaria do Estado de Saúde, 1976.

JARDIM, M. A. G; MOURÃO, L., GROSSMANN, M., Açaí (Euterpe oleracea Mart.): possibilidades e limites para o desenvolvimento sustentável no estuário Amazônico. Pará: Editora Museo Paraense Emilio Goeldi, 2004.

KOMATSU. T. R.; BURITI. F. C. A.; SAAD. S. M. I. Inovação, persistência e criatividade superando barreiras no desenvolvimento de alimentos probióticos. Revista Brasileira de Ciências Farmacêuticas. vol. 44, n. 3, jul./set., 2008.

RAUD. C. Os alimentos funcionais: a nova fronteira da indústria alimentar. Análise das estratégias da Danone e da Nestlé no mercado brasileiro de iogurtes. Revista de sociologia e política vol. 16, n. 31, p. 85-100, Nov. 2008

RAPACCI, M.; Apostila do VI Encontro Regional Sul de Ciência e Tecnologia de Alimentos. Leites Fermentados. Agosto 1999.

TENCHINI DE MACEDO, N.L.; Apostila Tecnologia de Fabricação de Leites Fermentados. Instituto de Laticínios Cândido Tostes. Novembro 2000.

INDUSTRIA DE LATICINIOS. Nova legislação de produtos lácteos e de alimentos pra fins especiais. Fonte Comunicações e Editora. São Paulo. 1998. 